\title{
Effects of Spatial Learning and Characteristics on Completing Spatial Tasks
}

\begin{abstract}
Shinta Wulandari ${ }^{1 *}$
${ }^{1}$ Mathematic Education Department, University of Borneo Tarakan

Corresponding author*Email: pmatshintaw@gmail.com

ABSTRACT

The purpose of this study is to describe the effects of students' spatial abilities and the characteristics of students' spatial ability in completing geometry tasks through learning interventions that emphasize spatial activity. This study employs a one-group pretest-posttest design. The research subjects were 33 grade VIII junior high school students. The learning process highlighting spatial activities was developed through three phases: initial investigation, prototyping, and assessment. Results show that students predominantly trusted in the physical features of spatial objects, i.e. the shape and positions also used the help of real objects as a reference for abstract concepts and took advantage of hand gestures when conducting spatial activities. Through intervention, students were trained to recognize spatial objects through their position, form, size, and the mental movement of spatial objects to determine the shape from a particular perspective. Findings further exhibit that there are relations between spatial ability and language ability. This research is relevant for teachers and students in the learning process of geometry that focuses on spatial activities, with steps of information acquisition, facilitation of spatial activity, reinforcement, and integration of information.
\end{abstract}

Keywords: spatial abilities, student strategies, geometry, spatial visualization, spatial orientation, spatial relations.

\section{INTRODUCTION}

Spatial abilities are cognitive processes in capturing, encoding and manipulating spatial shapes [1]. An individual with high spatial ability would have the capability to create a mental image of unseen spatial objects. The individual would be able to manipulate, imagine its movements, and determine the appearance of spatial shapes that are seen from a particular point of view. Several researchers proposed varied categorization of spatial components. In this research, three categories of spatial components followed are visualization, relations, and orientation [2], [3].

Mathematics and spatial ability have strong correlations. Studying geometry objects (i.e. two dimensions and three dimensions) requires spatial skills to understand them [4]. Specifically explained that understanding cube nets required spatial skills so that they can determine the relationship of cube nets with their cubes and the relationships between cube nets that are congruent. Other research states that in completing geometrical reasoning tasks, the use of spatial intuition is very important [5].

An understanding of the relationships and properties of spatial objects in geometry is needed in solving mathematical problems and problems related to spatial objects in everyday life [2], [3], [6]. But in reality, there are difficulties for students in solving spatial questions [7]. The difficulty that occurs is when students in grade VII high school solve problems that require the ability to imagine spatial objects that are viewed from a particular perspective or called the ability of spatial orientation. Most students do not change their point of view so that the appearance of the object represented does not represent the view that it should. For example, students state that a cube that is seen perpendicularly from above is in the form of a parallelogram, whereas if he changes his point of view it will look square. Another study also revealed that students in grades VII-IX in Japan have 
difficulty in giving reasons correctly to questions that contain spatial representations [4].

In school learning, geometric learning steps that contain spatial activities are needed. As stated by Cheng and Mix (2014) that students' mathematical abilities will be better if learning is designed to contain spatial activities. Other studies also convey that with spatial training, students' geometric abilities will increase [6], [8]-[10].

Learning geometry in Indonesia has not fully emphasized spatial activity. This influences students who have difficulty in solving problems that require spatial ability. Through this research, information about the effects of Geometry learning using spatial activity and characteristics of students' spatial abilities in grade VIII can be obtained in completing spatial tasks..

\section{METHOD}

\subsection{Settings and Participants}

This research was conducted in grade VIII students in secondary school consisting of 33 students. Learning material based on the applicable mathematics curriculum in Indonesia is to build flat side spaces. During spatial learning, students are guided by class teachers using learning steps that emphasize spatial activity. Participants have ages between 13 to 14 years. Learning is done in three meetings in three weeks, each meeting consisting of 80 minutes.

\subsection{Design}

This study uses a one-group pre-test post-test design research design to find out how much the effect of spatial learning on students. There are three steps to data collection. First, each student is given a pre-test in the form of a geometry task that emphasizes the spatial component. Second, each student receives an intervention in the form of spatial learning with spatial learning steps. Third, each student is given a post-test in the form of a geometry task that emphasizes spatial ability. Pre-test and post-test have different contents but are developed from indicators of spatial ability. After being given a pre-test and post-test, the results are then analyzed to find out the effects of spatial learning on students' spatial ability. To find out the characteristics in completing spatial tasks, an information search about strategies for completing spatial tasks is done during learning and when completing spatial tasks. In this case, a recording is done using a video while students complete the task and during spatial learning.

\subsection{The Instrument}

Instruments of spatial ability are arranged by linking geometry with spatial ability. The spatial ability instrument used in this study was validated by four experts and two practitioners.

Questions on spatial ability assessment are arranged based on three components of spatial ability, namely spatial visualization, spatial relations, and spatial orientation. Questions are presented in the format of short answers and answers that require explanation. The choice of reasoning format for the answer is used to find out how students use the term geometry to explain spatial questions.

\subsection{Data Collection and Analysis}

Spatial ability data is obtained by assigning spatial ability tasks to students who have participated in spatial learning. The task completion time is forty minutes. After being completed by students, the next instrument was analyzed. First, the work of students is coded, and the accuracy of the answer is measured using guidelines for scoring. Second, variations on the problem-solving strategy are described and supported by work results and documented through photos and videos. Subsequently classified the results of work and student strategies in completing spatial tasks. Each figure should have a caption. The caption should be concise and typed separately, not on the figure area; If figures have parts (for example, A and B), make sure all parts are explained in the caption.

\subsection{The Intervention}

Interventions in research services to provide students with learning experiences with spatial activities. Intervention in the form of learning geometry material with spatial activities with steps, namely: information acquisition, spatial activity facilitation, information reinforcement, and integration. After the third meeting given a spatial task. The steps of learning have been measured by the validator experts and practitioners so that this learning step is feasible to be used in spatial learning. The level of validity in the learning step is 0.87 which is in the high validity category.

\section{RESULTS}

\subsection{Quantitative Results}

In this section, the students' spatial ability test results were compared before and after the intervention using spatial learning. The test results are presented in the form of a percentage obtained from the comparison of correct answers and overall questions. This section does not require statistical tests because the two classes are not randomly selected. 
Table 1. Pretest and Posttest Results

\begin{tabular}{|c|c|c|}
\hline Task & Pretest (\%) & Posttest (\%) \\
\hline Spatial Visualization & 83 & 91 \\
\hline Spatial Relation & 65 & 86 \\
\hline Spatial Orientation & 24 & 44 \\
\hline
\end{tabular}

Table 1 shows that there was an increase in spatial ability after participating in spatial learning. The increase that occurred was $20 \%$. Based on Table 1, the results show that the achievement of spatial ability in spatial visualization is better than spatial relations and orientation.

\subsection{Analysis of Student Strategies in Solving Geometry Problems}

This section presents an analysis of students' strategies for completing spatial ability tests. Strategy comes from students' answers on the components of visualization, relations, and spatial orientation. Each component is given four questions for spatial visualization, every two questions in relation and spatial orientation. In each component was described, illustrated through pictures, and counted the number of students who answer according to criteria.

\subsubsection{Spatial Visualization}

This section presents an analysis of students' strategies for completing spatial ability tests. To find out students 'strategies in completing geometries assignments, the information is obtained through students' answers especially in the visualization, relations, and spatial orientation components. Each component is given four questions for spatial visualization, every two questions in relation and spatial orientation. In each component was described, illustrated through pictures, and counted the number of students who answer according to criteria.

Analysis on spatial visualization is done both on students' correct answers and wrong answers. Categorizing the spatial visualization problem-solving strategy consists of imagining, comparing directly, and using hand movements.

In the imagining strategy, students immediately guess answers without being seen using their five senses. Students who use this strategy can mentally imagine the position of a spatial object. For example, when asked to determine the position of the front side of the block with the backside known, students can immediately guess the position of the front side just by looking at the image of the block net that has been given to the task. This strategy is carried out by most students both before the intervention and after the intervention which is around $53 \%$.
The strategy of comparing directly appears to students by using models of tangible objects that are around them. For example, female students take paper, draw a net of cubes according to the pattern on the problem, cut out according to the pattern, mark the sides as caps, fold the webs to form a cube, find the sides as the base of the cube, mark the base of the cube, reopen the cube into nets cube net and finally answer the test questions by marking according to what is on the paper model. This strategy is not much done by students when solving problems related to cube nets that are $3 \%$ of the total students.

The strategy of using hand movements appears to students who move their palms in such a way as if they would form sides of a cube or blocks from the nets provided. For example, students determine the front side of a block through a drawing of a block of nets that has been determined behind it.

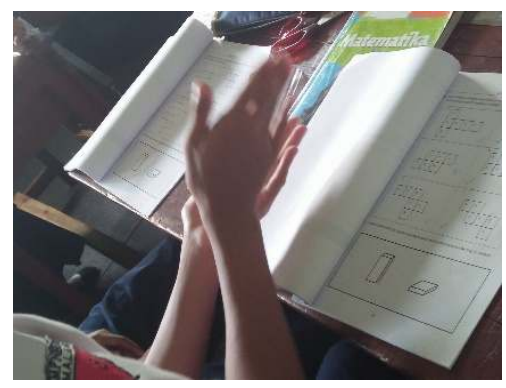

Figure 1. The hand as a representation of the folded side of the net

As seen in Figure 1, students place their hands in a side position on the block. Then the student moves the palm as a representation of the folded side, moves the palm of the other hand to the other side, remembers the position of the hand as the backside, determines the front side by remembering the palm that acts as the front side, and answers the questions according to the image. There are $44 \%$ of students who use this strategy.

\subsubsection{Spatial relations}

In solving the problem of spatial relations, there are two categories of strategies, namely connecting through the nets without paying attention to the images on the sides and relating the nets about the position and shape of the images. The first strategy is done by students to solve problems that relate to whether there is a match between the nets with a solid shape. Students who use the first strategy, begin to finish by determining whether the nets have a matching number of sides, side positions, and whether they can form solid shapes as intended in the problem. Then after finding out the nets are suitable; students immediately determine that there is a match between the nets with the intended building space without paying attention to the pictures on each side. 
While students complete with the second strategy, after knowing the relationship between the net and the solid shape, the students proceed by checking the images on the sides. Checking the image by looking at the shape of the image whether a circle, triangle, or parallelogram, then determine the direction of the image if the nets have been folded to form solid shapes.

After this spatial relation task, students are asked to explain the reasons why answering in such away. For example, in the problem of determining the relationship between images of cube nets with images of different numbers on the sides with cube images that contain figures are not related meaning cube nets do not form a cube-like in the picture in the problem. Most students answered correctly but when asked to write down reasons, most students did not mention the accuracy of the position of the image and the shape of the numbers. Students only check the shape of the net.

\subsubsection{Spatial Orientation}

The students' settlement strategies on spatial orientation questions are categorized into three namely egocentric and following the point of view. Students who use egocentric strategies show that they mentally do not change their point of view to be perpendicular to the object being observed. Students with egocentric strategies will imagine according to what appears in front of them without changing the point of view as requested in the problem. For example, students are asked to paint shapes because of imagining a cube that is viewed from an upright angle. Students who use egocentric strategies solve problems by imagining the cube as shown in the picture in the problem. Then he imagined the top side but with a viewpoint that was perpendicular to the front side, so the student decided that the top side was in the form of a parallelogram and he painted the parallelogram shape as an answer to that question.

Student strategy in the category according to the point of view is the strategy of students who imagine the spatial object by the requested perspective. For example, students are asked to determine the appearance of a block of a certain size seen from the right side. To answer the question, students in the appropriate category viewpoint will see a picture with visible features, imagine if they are on the right side of the block perpendicularly, determine its rectangular appearance according to the size given to the problem.

\section{DISCUSSION}

The effects and characteristics of spatial abilities are discussed in this section.

\subsection{The Effects of Spatial Activities}

Students who follow learning with spatial activity on the material properties of cubes and blocks are allowed to explore the cube and block models to get information on objects of size, shape from various points of view and their relationships with other parts. By interacting directly with learning media such as cube and box models or other manipulative objects can help students identify objects well [11]-[13].

Information obtained from exploring cube and block models can be identified by the terms and concepts students have as material for associating with new knowledge. The terms and concepts obtained are parts of the cube and blocks, including sides, edges, and vertices. To find out the terms and concepts students have, a class discussion is conducted by asking group representatives to present the terms and concepts they have. Then based on class discussion the teacher gives a response to the results of the class discussion, if the concepts and terms are correct then the learning continues to the next activity. If there are still errors, then the teacher corrects directly then continues to the next activity. The choice of terms is necessary for spatial learning because in spatial representation it is known through appropriate terms [14]

Based on the terms and concepts of the discussion results, then students are ready to obtain new knowledge. The next student activity is to practice spatial abilities and has been pre-designed by the teacher. In the matter of the nature of the cube and the blocks, spatial activities are 1) sketching the cube after observing it before, 2) determining the characteristics of the cube based on the sketch made, 3 ) determining the size of the cube based on other known sides, 4) determining the relation of two edges that are parallel, intersect and intersect, 5) the appearance of cubes and blocks that are seen perpendicularly from a certain direction. Through activities that train spatial ability, it is hoped that it can improve students' spatial abilities as conveyed by Cheng \& Mix (2014) that spatial training can improve spatial abilities so that their mathematical abilities will also increase.

To further strengthen the information learned, students work on the exercises provided on the worksheet. Giving challenging assignments to the exercises will make someone remember the material that has been learned and organize information so that information can be stored properly [15], [16].

Spatial learning ends with an integration activity in which in this activity, students are asked to express their opinions about what has been learned at the meeting. Then the teacher gives a conclusion based on the results of student reflection. Reflecting and concluding activities help students in their learning process. 
Before participating in spatial learning, students have difficulty when determining the position of parts of cubes and blocks, recognizing the characteristics of net cubes and blocks, determining the appearance of cubes and beams seen at certain angles, and the relationship between cubes and blocks to the net. After receiving spatial learning, students can: 1) state the position of parts in cubes and blocks, 2) construct and represent the shapes of cubes and blocks, 3) determine the relationship between parts of cubes and blocks, and 4) draw the shape or position of a cube and blocks that are seen from a certain point of view.

\subsection{Characteristics of Spatial Ability of grade VIII}

Various strategies are shown by students in solving spatial problems. Most students can solve spatial visualization problem types correctly using the strategy of imagining and using hand movements. The strategy used by students in solving spatial relation questions is to use a picture of a net without paying attention to the image on the side of a drawing of a room. While the strategy on spatial orientation used by most students is following the point of view. Thus, apart from interventions in the form of spatial learning, it can be stated that students having spatial abilities have the potential to be further improved especially in spatial orientation activities.

After following a spatial learning intervention for two meetings or more than one week, students experience an increase in spatial ability both in visualization, relations, and spatial orientation. The increase is thought to be due to an intervention in the form of spatial learning which includes activities that train students how to observe spatial objects from their position, shape, size, and displacement. Spatial learning trains students to observe spatial objects and then see from a certain point of view so that when spatial objects are not in front of them, students can imagine the object and can mentally imagine its shape when viewed from a particular viewpoint. Spatial learning trains students to determine the relationship between two spatial objects both spatial objects in space and between parts of a spatial object.

Characteristics of students' spatial abilities based on this study are more dominant in spatial visualization. That is, in solving spatial problems students better understand how to recognize the shape and position of a spatial object. Difficulties arise when spatial objects are displayed in more complex shapes and the object is shifted, rotated, and viewed in a particular direction of view. Spatial problems solving strategies mostly use the Imaginary approach (visual imagery) wherein the strategy students begin by encoding, imagining, and representing. In the process of imagining, there are those without object assistance, using the help of tangible object models and using palm movements. The results of this study are following Cohen \& Hegarty (2014), that in completing sliced tasks in building space, the subject is more dominant using an imaginary approach than an analytic approach.

\section{CONCLUSION}

This study describes that the students of class VIII have dominant spatial visualization ability when solving geometrical tasks. Spatial information given on the geometrical objects is more frequent to be obtained from the physical characteristics and the display from a certain angle. The students' problem-solving abilities on spatial relations have also improved significantly after participating in the spatial intervention. Through spatial activities, the students enriched their term collections to state relations between spatial objects. Also, regarding spatial orientation, the students tend to be included in the egocentric type, who tend to preserve the angle of objects as being asked in the test items. As a consequence, an incongruity between the real display and the mental display took place. After participating in the intervention, the students' spatial orientation was improved. Through activities stimulating students to start with an interaction with different models, students were trained to identify the display of objects from a particular point of view and to represent it. However, overall, students' spatial orientation was still substandard after the intervention. In this research, students were given interventions in the form of spatial learning. The activities encourage students to explore objects, digging up terms to describe objects, and determine the display of an object from certain angles.

Implications of this research are, determining that students in junior high schools can finish spatial problems, especially on test items requiring spatial visualization abilities. Students' spatial ability could be trained by designing a learning process aiming at elevating students' spatial ability. Findings on this research claimed that spatial learning should be started with spatial information acquisition to provide students with formal terms and concepts in geometry. The mastery of the concepts is required in representing a spatial object both verbally and in the form of narration. It is concluded that in the geometry learning process, activities training students to dig up spatial information, to represent spatial information, and to solve the task accurately.

Future research should be conducted towards more magnitude numbers of subjects to dig up the effect of spatial learning on junior high school students' spatial abilities, particularly in Indonesia. Specifically, further research should elaborate on the development of learning design, instruments, and tools to improve students' spatial abilities. 


\section{REFERENCES}

[1] N. S. Newcombe, "Thinking spatially in the science classroom," Curr. Opin. Behav. Sci., vol. 10, pp. 16, 2016, doi: 10.1016/j.cobeha.2016.04.010.

[2] Y. Wakabayashi and T. Ishikawa, "Spatial thinking in geographic information science: A review of past studies and prospects for the future," Procedia - Soc. Behav. Sci., vol. 21, pp. 304-313, 2011, doi: 10.1016/j.sbspro.2011.07.031.

[3] H. B. Yilmaz, "On the development and measurement of spatial ability," Int. Electron. J. Elem. Educ., vol. 1, no. 2, pp. 83-96, 2009.

[4] T. Fujita, Y. Kondo, H. Kumakura, and S. Kunimune, "Students' geometric thinking with cube representations: Assessment framework and empirical evidence," J. Math. Behav., vol. 46, pp. 96-111, 2017, doi: 10.1016/j.jmathb.2017.03.003.

[5] D. Küchemann and C. Hoyles, "and Patterns in Its Development: Insights," Int. J. Sci. Math. Educ., vol. 4, no. 4, pp. 581-608, 2006.

[6] J. Wai, D. Lubinski, and C. P. Benbow, "Spatial Ability for STEM Domains: Aligning Over 50 Years of Cumulative Psychological Knowledge Solidifies Its Importance," J. Educ. Psychol., vol. 101, no. 4, pp. 817-835, 2009, doi: $10.1037 / \mathrm{a} 0016127$.

[7] S. Wulandari, C. Sa'Dijah, E. B. Irawan, and I. M. Sulandra, "Spatial information processing of seventh grader in solving Geometry problems," IOP Conf. Ser. Earth Environ. Sci., vol. 243, no. 1, 2019, doi: 10.1088/1755-1315/243/1/012135.

[8] T. Lowrie, D. Harris, T. Logan, and M. Hegarty, "The Impact of a Spatial Intervention Program on Students' Spatial Reasoning and Mathematics Performance," J. Exp. Educ., vol. 89, no. 2, pp. 259277, 2021, doi: 10.1080/00220973.2019.1684869.

[9] W. Möhring, N. S. Newcombe, and A. Frick, "The relation between spatial thinking and proportional reasoning in preschoolers," J. Exp. Child Psychol., vol. 132, pp. 213-220, 2015, doi: 10.1016/j.jecp.2015.01.005.

[10] D. H. Uttal, D. I. Miller, and N. S. Newcombe, "Exploring and Enhancing Spatial Thinking: Links to Achievement in Science, Technology, Engineering, and Mathematics?," Curr. Dir. Psychol. Sci., vol. 22, no. 5, pp. 367-373, 2013, doi: $10.1177 / 0963721413484756$.

[11] Y. P. Tam, T. T. Y. Wong, and W. W. L. Chan, "The relation between spatial skills and mathematical abilities: The mediating role of mental number line representation," Contemp. Educ. Psychol., vol. 56, no. October 2018, pp. 14-24, 2019, doi: 10.1016/j.cedpsych.2018.10.007.

[12] M. H. Fischer, M. Riello, B. L. Giordano, and E. Rusconi, "Singing numbers... in cognitive space - a dual-task study of the link between pitch, space, and numbers," Top. Cogn. Sci., vol. 5, no. 2, pp. 354366, 2013, doi: 10.1111/tops.12017.

[13] E. V. Laski and R. S. Siegler, "Learning from number board games: You learn what you encode," Dev. Psychol., vol. 50, no. 3, pp. 853-864, 2014, doi: $10.1037 / \mathrm{a} 0034321$.

[14] N. K. Simms and D. Gentner, "Finding the middle: Spatial language and spatial reasoning," Cogn. Dev., vol. 50, no. November 2018, pp. 177-194, 2019, doi: 10.1016/j.cogdev.2019.04.002.

[15] C. Grimsen, H. Hildebrandt, and M. Fahle, "Dissociation of egocentric and allocentric coding of space in visual search after right middle cerebral artery stroke," Neuropsychologia, vol. 46, no. 3, pp. 902-914, 2008, doi: 10.1016/j.neuropsychologia.2007.11.028.

[16] L. Wang and M. Carr, "Working Memory and Strategy Use Contribute to Gender Differences in Spatial Ability," Educ. Psychol., vol. 49, no. 4, pp. 261-282, 2014, doi: $10.1080 / 00461520.2014 .960568$. 